\title{
Air Traffic Flow Forecast in the Period of Major Events Based on Cost-Effectiveness Optimization
}

\author{
Sofia Storozhenko, Andrey Popov, Vladimir Sikachev \\ State Scientific Research Institute of Aviation Systems \\ 7 Victorenko str., Moscow, Russia \\ storozhenko@gosniias.ru; andey.popov@gosniias.ru; vov@gosniias.ru
}

\begin{abstract}
Major events, for example Olympic Games or FIFA World Cup, are very popular with people from all over the world. The demand for air travel noticeably rises at these periods. In that case, research has to be carried out in order to forecast the traffic flow to make all members of ATM system (airlines, airports, ATC and etc.) ready for it. In this paper the method to figure out the possible structure of air traffic flows will be described. This method also can be used to estimate the optimal aircraft fleet to transport the additional passengers or the optimal usage of available aircrafts. The focus will be on minimizing airline's expenses. The main goal of this study is to transform the task of people transportation into math problem. The way to state optimization problem will be represented. This paper also considers different types of research conditions and suitable constraints for each of them.
\end{abstract}

Keywords: cost minimization, air traffic flow structure, major events, forecasting

\section{Introduction}

Major events influence transportation system a lot especially in countries they are taking places in. Important information needed to forecast the influence on ATM system is the structure of air traffic flow. In other words the directions where flights would be taken and the type of aircrafts which would be used should be predicted.

On the other hand, it may be useful for airlines to estimate what aircraft types are more effective to use in this period of time. In this paper it is taken that the main criterion is the minimization of airline's expenses.

According to these aspects the method of predicting air traffic flow structure is suggested in this paper. The prediction can be divided in five parts:

- Transport network construction.

- Formal optimization problem statement.

- Handling of optimization problem.

- Schedule construction.

- Merger with scheduled flights.

In this paper first two steps will be considered in details while for the last three steps general recommendation will be given, as the method should be used strongly depends on research goals. Moreover, the last two steps may be skipped if there is no need in estimating the influence on ATM system.

\section{Transport network}

\subsection{Input}

To form the transport network the following information is needed:

- The set of cities where the significant passenger flow is expected from (set of Sources, $P$ ).

- The set of cities where the significant passenger flow is expected to (set of Stocks, $Q$ ).

- The set of cities which can be used as hubs (set of Hubs, $H$ ). In this study it is taken that passengers can reach their destination with no more than 2 transfers in Hubs 

$C_{p q}$.

- The number of passengers which are expected to be transported from each Source $(\forall p \in P)$ to each Stock $(\forall q \in Q)$,

It is important to note that objects from $H$ may have functions of Source or Stock or both of them but still will be referred to set $H$ (not $P$ or $Q$ ).

Also to evaluate expenses the following data is needed:

- Aircraft performance, airspace structure and the model for route construction.

- The cost of airports services and fuel.

- Types of aircraft which are available (set of aircraft types, $L$ ).

\subsection{Transport network construction}

The transportation net will be presented as a set of directed weighted graphs (for each available aircraft type). The set of vertices consists of defined cities (Sources, Stocks, Hubs). Hereinafter, letters $i, j$ will be used to define any vertex. In other words, within this paper $i, j \in H \cup P \cup Q$.

The weight for each edges is determined by the cost of flight between it vertices. The definition of cost mostly depends on the researchers. In this paper fuel and airports expenses are taken for cost. To evaluate fuel consumption was used the model of route construction based on method described here [1].

If aircraft is unable to make a flight between two vertices (too long distance or one of the airports is unable to service this type of aircraft), the cost is taken infinitely large.

The cost of flight on each edge $(i, j)$ will be defined as a "cost" vector (1), where $l$ is an number of aircraft type from $L$. If $\zeta_{i j}^{l}$ is the expense of the flight of type $l$ on the edge $(i, j)$, then:

$$
\overrightarrow{\zeta_{l j}}=\left\{\zeta_{i j}^{l}\right\}_{l=\overline{1,|L|}}
$$

Also for each type has to be determined the biggest number of passengers that can be transported, a "seet" vector (2). If $\xi^{l}$ the number of passengers that can be transported by aircraft of type $l$, then:

$$
\vec{\xi}=\left\{\xi^{l}\right\}_{l=\overline{1,|L|}}
$$

If needed, a "seet" vector can be defined on each edge separately.

\section{Optimization problem statement}

\subsection{Variables}

In this paper the number of used aircraft and the number of transported passengers are used as variables the loss function is minimizer over.

The number of used aircraft on each edge $(i, j)$ will be defined as a "use" vector, $\overrightarrow{x_{l \jmath}}$. If $x_{i j}^{l}$ is the number of used aircrafts of type $l$ on edge $(i, j)$, then the "use" vector can be determined as (3):

$$
\overrightarrow{x_{l j}}=\left\{x_{i j}^{l}\right\}_{l=\overline{1,|L|}}
$$

The number of passengers on each edge $(i, j)$ from each direction (pair Source - Stock) is defined as $y_{i j}^{p q}$, where $p \in P \cup H, q \in Q \cup H$ (as Hub can be used as Source or Stock).

\subsection{Loss function}

The loss function is strongly depends on the research goals. In approbation the linear loss function (4) was used. To take into account the airport payments for each passenger, the cost of them was added as $\zeta_{i j}^{*}$.

ICTE 120-2 


$$
f(\vec{X}, y)=\sum_{i, j}{\overrightarrow{x_{\imath j}}}^{T} \overrightarrow{\zeta_{\imath \jmath}}+\sum_{\substack{i, j \\ p \in P \cup H \\ q \in Q \cup H}} \zeta_{i j}^{*} y_{i j}^{p q}
$$

Where

$$
\begin{gathered}
\vec{X}=\left\{\overrightarrow{x_{\imath \jmath}}\right\}_{i, j \in P \cup H \cup Q} \\
y=\left\{y_{i j}^{p q}\right\}_{\substack{p \in P \cup H \\
q \in Q \cup H}}
\end{gathered}
$$

The optimal resolution is $\left(\overrightarrow{X^{*}}, y^{*}\right)$, which :

$$
f\left(\overrightarrow{X^{*}}, y^{*}\right)=\min _{\vec{X}, y}\left(\sum_{i, j}{\overrightarrow{x_{l j}}}^{T} \overrightarrow{\zeta_{l j}}+\sum_{\substack{i, j \\ p \in P \cup H \\ q \in Q \cup H}} \zeta_{i j}^{*} y_{i j}^{p q}\right)
$$

\subsection{Constraints}

There may be different types of constraints in this problem. Here the essential ones will be shown and several examples of specific constraints will be given.

The first group ((5) and (6)) of constraints guarantees the necessary number of passeng0ers leave their Sources (for their Stocks or Hubs) and the necessary number of passengers arrive at their Stocks (from their Sources or Hubs).

$$
\begin{array}{ll}
\forall p \in P, \forall q \in Q: & \sum_{j \in H \cup Q} y_{p j}^{p q} \geq C_{p q}-{\overrightarrow{x_{p q}}}^{T} \vec{\xi} \\
\forall p \in P, \forall q \in Q: & \sum_{i \in P \cup H} y_{i q}^{p q} \geq C_{p q}-{\overrightarrow{x_{p q}}}^{T} \vec{\xi}
\end{array}
$$

The next group of constraints (7) guarantees the balance in Hubs (that the number of passengers from each direction who have arrived at each Hub will be not less than the number of passengers who left).

$$
\forall p \in P \cup H, \forall q \in Q \cup H, \forall h \in H: \quad \sum_{i \in P \cup H} y_{i h}^{p q} \geq \sum_{j \in H \cup Q} y_{h j}^{p q}+C_{p q}^{h}
$$

where

$$
C_{p q}^{h}=\left\{\begin{array}{cc}
0, & p \neq h, q \neq h \\
-C_{h q}, & p=h \\
C_{p h} & q=h
\end{array}\right.
$$

The last group of constraints (8) provides enough aircraft to transport all passengers from all directions on every edge. 


$$
{\overrightarrow{x_{l j}}}^{T} \vec{\xi} \geq \sum_{\substack{p \in P \\ q \in Q}} y_{i j}^{p q}
$$

Constraints written above ensure the correctness of the result. In other words, they guarantee that all passengers will be transferred to their destination in a right way. What is more, the obtained vector $\vec{X}$ is the optimal set of aircraft with minimized airline's expenses. It can be useful to estimate the optimal aircraft fleet needed to transfer all passengers.

When the information about the available aircraft fleet size has to be taken into consideration, more constraints are needed. In this paper two examples of possible limits are given.

The first one is to use all of available aircraft (type and number are known) with the expenses minimizing.

The second one is to get an optimal usage of aircraft fleet when we know where each of aircraft is at the beginning of the transportation (the type and number of aircrafts in each airport are known).

The number of available aircraft of type $l$ is defined as $\theta^{l}$, the number of available aircraft of type $l$ in vertex $i$ is defined as $\theta_{i}^{l}$.

The constraints used to satisfy the first condition are following:

$$
\begin{aligned}
& \forall l \in L: \sum_{\substack{p \in P \\
j \in H \cup Q}} x_{p j}^{l} \leq \theta^{l} \\
& \forall l \in L: \sum_{\substack{u \in H \\
w \in H}} x_{u w}^{l} \leq \theta^{l}-\sum_{\substack{p \in P \\
q \in Q}} x_{p q}^{l} \\
& \forall l \in L, \forall h \in H: \sum_{u \in H} x_{h u}^{l} \leq \theta^{l}-\sum_{\substack{p \in P \\
j \in H \cup Q}} x_{p j}^{l} \\
& \forall l \in L: \sum_{\substack{u \in H \\
q \in Q}} x_{u q}^{l} \leq \theta^{l}-\sum_{\substack{p \in P \\
q \in Q}} x_{p q}^{l \neq h} \\
& \forall l \in L, \forall h \in H: \quad \sum_{q \in Q} x_{h q}^{l} \leq \theta^{l}-\sum_{\substack{p \in P \\
j \in H \cup Q \\
j \neq h}}^{q \in Q} x_{p j}^{l}-\sum_{\substack{u, w \in H \\
w \neq h}} x_{u w}^{l}
\end{aligned}
$$

The constraints used to satisfy the second condition are following:

$$
\begin{gathered}
\forall l \in L: \sum_{j} x_{p j}^{l} \leq \theta_{p}^{l}, \forall p \in P \\
\forall l \in L: \sum_{u \in H} x_{h u}^{l} \leq \theta_{h}^{l}+\sum_{p \in P} x_{p h}^{l}, \forall h \in H \\
\forall l \in L: \sum_{q \in Q} x_{h q}^{l} \leq \theta_{h}^{l}+\sum_{i \in P \cup H} x_{i h}^{l}-\sum_{u \in H} x_{h u}^{l}, \forall h \in H
\end{gathered}
$$

The study shows that this problem is very flexible and can be modified by changing loss function and adding constraints. 


\section{Algorithms, schedule and constructing flow for research}

The algorithm for solving optimization problem strongly depends on the appearance of loss function and additional constraints. In this paper all given relationships are linear so the methods of linear programming are applicable (Simplex algorithm). But the difficult is that variables have to be integer. In that case Gomory's cutting-plane method, "branch and bound" and other algorithm for solving integer linear programing problems can be used. Also different math tools afford functions for handling such problems.

To construct schedule also different methods can be used. The authors find it suitable to use the modification of method called "List Scheduling", described here [2].

If the forecasted structure is required for ATM system research the obtained air traffic flow has to be merged with the real or forecasted scheduled flights. For this needs methods of constructing stochastic flows with predicted intensity can be used (described here [3]).

\section{Results}

The model based on described method was integrated into KIM OrVD (Air traffic management research simulation tool) [3]. It is used for preparing air traffic flow for fast-time simulation.

The optimization problem resolution for the transportation net with several aircraft types and cities is very hard to be presented in a clear view because of its complexity.

In that case, the resolution for the simplest example will be given in details to illustrate the functioning of the method.

The input data is shown in table 1 .

Table 1: Input data about additional passenger flow and available aircrafts.

\begin{tabular}{|c|c|c|c|}
\hline & Saint Petersburg & Moscow & Volgograd \\
\hline \multicolumn{4}{|c|}{ Function } \\
\hline & Source & Source, Hub & Stock \\
\hline \multicolumn{4}{|c|}{ Passenger flows } \\
\hline To Volgograd & 1200 & 1000 & - \\
\hline \multicolumn{4}{|c|}{ Available aircraft fleet (in airports) } \\
\hline Medium (185 seets) & 10 & 0 & - \\
\hline Heavy (580 seets) & 10 & 10 & 0 \\
\hline \multicolumn{4}{|c|}{ Available aircraft fleet (common) } \\
\hline Medium (185 seets) & \multirow{2}{*}{\multicolumn{3}{|c|}{$\frac{10}{10}$}} \\
\hline Heavy (580 seets) & & & \\
\hline \multicolumn{4}{|c|}{ Available aircraft types } \\
\hline \multicolumn{4}{|c|}{ Medium (185 seets) } \\
\hline \multicolumn{4}{|c|}{ Heavy (580 seets) } \\
\hline
\end{tabular}

It has to be noted that these two types of aircraft was chosen to illustrate the functionality.

The results for each problem are given in tables 2-4.

Table 2: Optimal aircraft use.

\begin{tabular}{|c|c|c|c|c|}
\hline Edge & Aircraft type & Optimal solution & Common fleet & Fleet in airports \\
\hline $\begin{array}{c}\text { Saint } \\
\text { Petersburg }- \\
\text { Volgograd }\end{array}$ & Medium & 6 & 6 & 6 \\
\cline { 2 - 5 } $\begin{array}{c}\text { Saint } \\
\text { Petersburg }- \\
\text { Moscow }\end{array}$ & Heavy & 0 & 0 & 0 \\
\cline { 2 - 5 } & Medium & 1 & 0 & 0 \\
\hline $\begin{array}{c}\text { Moscow }- \\
\text { Volgograd }\end{array}$ & Medium & 0 & 3 & 0 \\
\cline { 2 - 5 } & Heavy & 0 & 1 & 2 \\
\hline
\end{tabular}

ICTE 120-5 
Table 3: Optimal passengers' distribution.

\begin{tabular}{|c|c|c|c|c|}
\hline Edge & Direction & Optimal solution & Common fleet & Fleet in airports \\
\hline \multirow{2}{*}{$\begin{array}{c}\text { Saint } \\
\text { Petersburg - } \\
\text { Volgograd }\end{array}$} & $\begin{array}{c}\text { Saint Petersburg - } \\
\text { Volgograd }\end{array}$ & 1110 & 1110 & 1110 \\
\hline & $\begin{array}{l}\text { Moscow - } \\
\text { Volgograd }\end{array}$ & 0 & 0 & 0 \\
\hline \multirow{2}{*}{$\begin{array}{c}\text { Saint } \\
\text { Petersburg - } \\
\text { Moscow }\end{array}$} & $\begin{array}{c}\text { Saint Petersburg - } \\
\text { Volgograd }\end{array}$ & 90 & 90 & 90 \\
\hline & $\begin{array}{l}\text { Moscow - } \\
\text { Volgograd }\end{array}$ & 0 & 0 & 0 \\
\hline \multirow{2}{*}{$\begin{array}{l}\text { Moscow - } \\
\text { Volgograd }\end{array}$} & $\begin{array}{c}\text { Saint Petersburg - } \\
\text { Volgograd }\end{array}$ & 90 & 90 & 90 \\
\hline & $\begin{array}{l}\text { Moscow - } \\
\text { Volgograd }\end{array}$ & 1000 & 1000 & 1000 \\
\hline
\end{tabular}

Table 4: Relative airlines expenses.

\begin{tabular}{|c|c|c|c|}
\hline & Optimal solution & Common fleet & Fleet in airports \\
\hline Relative airlines expenses & $100 \%$ & $104 \%$ & $109 \%$ \\
\hline
\end{tabular}

From table 2 it is seen that the best way to transport all passengers is to use only medium aircraft type and to send part of them with the transfer in Moscow (because if the additional aircraft has to be used it is better to send it to Moscow, as the distance is shorter while additional passengers will not increase the amount aircrafts which fly from Moscow to Volgograd). When the amount of available medium aircrafts is limited the heavy ones have to be used as there are not enough medium aircrafts. The use of heave aircrafts causes the increase of expenses.

In table 5 the results for transport network with several cities are represented. The table not full and show only number of passengers on several edges and the number of passengers who travel with the use of Hubs. The functions of cities are illustrated on figure 1. Here blue is for Sources, violet if for Hubs, red for Stocks. The Stocks are taken from the list of cities where the FIFA World Cup 2018 will be taking places in. Moscow and Yekaterinburg are Stocks and Hubs at the same time. To simplify, only one type of heavy aircraft is available. There is no limit on the number of aircrafts.

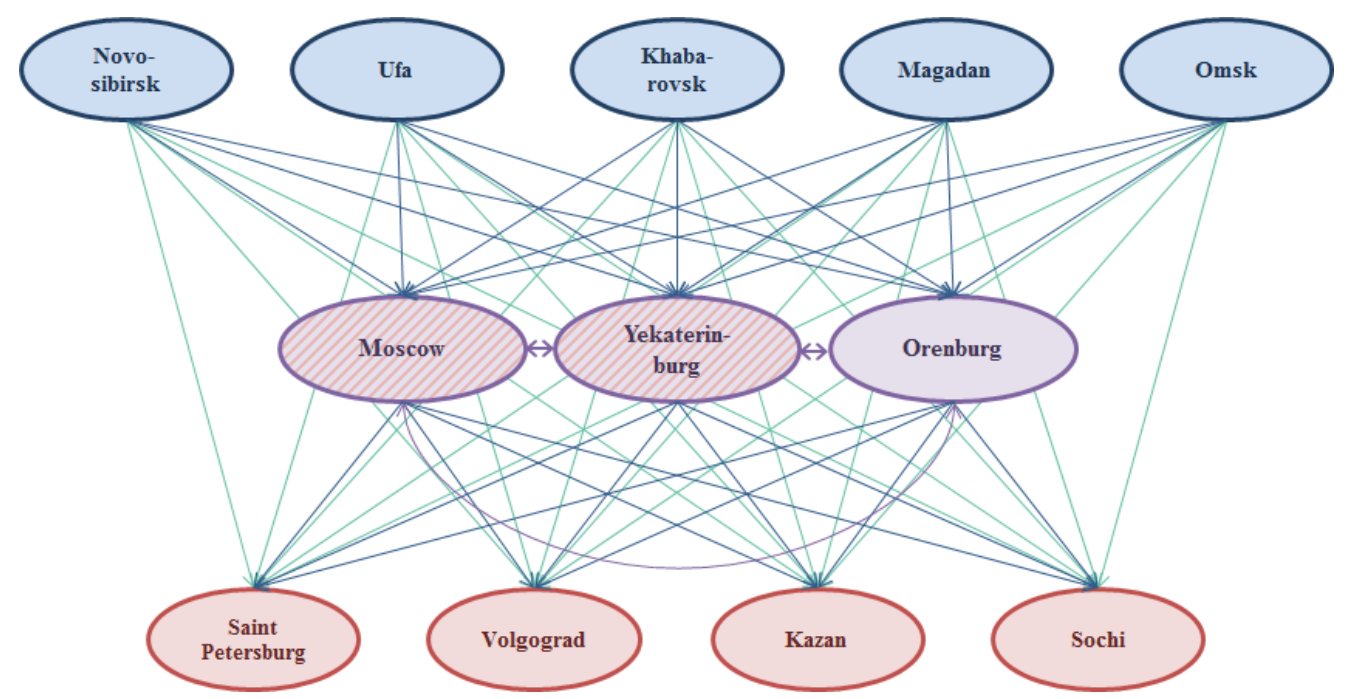

Fig. 1: Transport network. 
Table 5: Optimal passengers' distribution.

\begin{tabular}{|c|c|c|c|}
\hline Edge & Aircrafts used & Number of passengers & $\begin{array}{c}\text { Number of passengers who travel } \\
\text { with the use of Hubs }\end{array}$ \\
\hline Novosibirsk- Moscow & 1 & 420 & 220 \\
\hline Novosibirsk - Yekaterinburg & 1 & 480 & 380 \\
\hline Ufa - Moscow & 1 & 400 & 200 \\
\hline Ufa - Yekaterinburg & 1 & 500 & 400 \\
\hline Khabarovsk - Moscow & 2 & 720 & 320 \\
\hline Khabarovsk - Yekaterinburg & 1 & 380 & 280 \\
\hline Magadan - Yekaterinburg & 1 & 500 & 400 \\
\hline Omsk - Moscow & 1 & 200 & 0 \\
\hline Omsk - Yekaterinburg & 2 & 700 & 320 \\
\hline Moscow - Saint Petersburg & 1 & 320 & 420 \\
\hline Moscow - Sochi & 1 & 420 & 480 \\
\hline Yekaterinburg - Saint Petersburg & 1 & 480 & 500 \\
\hline Yekaterinburg - Volgograd & 1 & 500 & 500 \\
\hline Yekaterinburg - Kazan & 1 & 500 & 580 \\
\hline Yekaterinburg - Sochi & 1 & 580 & 0 \\
\hline Magadan - Saint Petersburg & 1 & 100 & \\
\hline
\end{tabular}

Table 5 shows that it is effective to "gather" passengers in Hubs and then send them to their Stocks.

\section{Conclusion}

The way to predict the air traffic flow structure during the period of major events is presented in this paper. The method described here is based on transport network. This network is used for discrete optimization problem statement.

The main criterion taken in this study is the minimization of airline's expenses. In this paper was given the example of the loss function, the essential constraint which ensures the correctness of the result. Moreover, two examples of constraint to take into consideration the number of aircrafts limitation were given.

This method appears to be flexible. The problem can be easily modified by adding extra constraint or changing the loss function.

This method also may be useful for research. It lets a researcher predict the structure of flow without going into details with airline's policy. Integrating this model into simulation tool gives additional opportunities for preparing input data for simulation.

\section{Acknowledgements}

This work was made within the modernisation of KIM OrVD. This tool was developed by State Scientific Research Institute of Aviation Systems.

We would like to thank people from our laboratory for help and advice, especially Larisa Vishnyakova.

\section{References}

[1] E. Filenkova, "Universal algorithm and program tools of 4D flight plan synthesis under conditions of dynamic special use airspace," in Proceedings of the $3^{\text {rd }}$ CEAS Air \& Space Conference, pp. 1117-1124.

[2] G. de Micheli, Synthesis and optimization of digital circuits. New York: McGraw-Hill, 1994.

[3] O. V. Degtiarev, V. P. Egorova, "Regulated Air Traffic Flow Simulation Tool," in Proceedings of 16th IFAC Symposium on Automatic Control in Aerospace. 